\title{
The Effect of the Parameters of a Vibration-Based Impact Mode Piezoelectric Power Generator
}

\author{
Amat A. Basari, ${ }^{1}$ S. Awaji, ${ }^{1}$ S. Sakamoto, ${ }^{1}$ S. Hashimoto, ${ }^{1}$ B. Homma, ${ }^{2}$ K. Suto, ${ }^{2}$ \\ H. Okada, ${ }^{2}$ H. Okuno, ${ }^{2}$ K. Kobayashi, ${ }^{2}$ and S. Kumagai ${ }^{2}$ \\ ${ }^{1}$ Division of Electronics and Informatics, School of Science and Technology, Faculty of Gunma University, 1-5-1 Tenjin-cho, \\ Kiryu, Gunma 376-8515, Japan \\ ${ }^{2}$ Research and Development Department, Mitsuba Corporation, 1-2681 Hirosawa-cho, Kiryu, Gunma 376-8555, Japan
}

Correspondence should be addressed to Amat A. Basari; amat@utem.edu.my and S. Hashimoto; hashimotos@gunma-u.ac.jp

Received 12 February 2015; Accepted 11 May 2015

Academic Editor: Nuno M. Maia

Copyright (C) 2015 Amat A. Basari et al. This is an open access article distributed under the Creative Commons Attribution License, which permits unrestricted use, distribution, and reproduction in any medium, provided the original work is properly cited.

\begin{abstract}
This study reports the effects of the parameters of a vibration-based impact mode piezoelectric power generator. First, an evaluation of the effects of the impact parameters, the mass, and the impact velocity is presented. It is found that the output voltage of the piezoelectric device in impact mode is directly proportional to the velocity, whereas the output power is equal to a quadratic function of the same variable. For the same impact momentum, the effect of the velocity in generating a higher peak output is dominant compared with the mass. Second, the vibration-based impact mode piezoelectric power generator is discussed. The experimental results show that a wider operating frequency bandwidth of the output power can be achieved with the preloading configuration. However, regarding magnitude, due to the high velocity of impact, the configuration with a gap between the tip and the piezoelectric device produces a higher output.
\end{abstract}

\section{Introduction}

Research advancements in mechanical vibration energy harvesting have been widely reported for decades. Energy from these harvesting systems is expected to be used to power low-power devices, such as LEDs, tire pressure monitoring systems, and many others. The objectives of the study are to propose a new design and to evaluate the factors that affect the output power generation. In general, mechanical vibration is converted to electrical energy using three types of devices: piezoelectric, electrostatic, and electromagnetic devices. Both analytical [1] and experimental analyses [2-5] have shown that there are various factors that affect the performance of such devices. The evaluation of each factor is very subjective that strongly depends on the devices, the environment, and the type of the vibration.

In the case of vibration energy harvesting using piezoelectric devices, for linear vibration motion, the basic operation of power generation can be divided into two modes: bending mode and impact mode. In bending mode for power generation using a piezoelectric cantilever beam, one end of the device is attached to the vibration sources and the other end freely vibrates with the sources of the vibration. To improve the output power of the piezoelectric power generator in bending mode, the shape of the device is critical, as it has been shown that devices of certain shapes are more effective than others $[6,7]$.

Another important factor for the optimum output is impedance matching $[8,9]$. However, impedance matching is dependent to the resonant frequency of the structures, which means that for low resonant frequency structures, a large matching impedance is required. These are among the important factors that have been considered in the design of vibration-based bending mode piezoelectric power generators.

In the case of vibration-based impact mode power generation, piezoelectric devices do not deform due the vibration. The deformation is due to the impact. As reported in [10], a structure with a freely moving steel ball repeatedly hits the piezoelectric wall to generate electricity. At 
the beginning of the design process, a weight drop experiment is conducted. The output power from this experiment is found to be relatively higher compared with the output power of the designated device. One suggestion regarding optimizing the output power is that the steel ball must be large and heavy. Another design of an impact mode piezoelectric power generator is reported by [11]. An impact mode power generator consisting of a vibrating beam with a piezoelectric device on top and two additional piezoelectric cantilever beams placed at each side of the vibrating beam is proposed. The vibrating beam has an extended rectangular tip on which a mass is fixed. When the beam vibrates, it hits both piezoelectric beams and, due to the impact, electricity is generated. The implementation target of the device is to harvest low frequency vibrations, such as human motionrelated movements. The optimization procedure is based on the impedance matching technique. Another analysis and discussion on the combination of a bending and impact mode power generator is reported in [12]. It is reported that, in terms of voltage, the bending mode piezoelectric specimen generates higher values than that of the impact mode piezoelectric specimen, although how the output can be optimized for the designated device is not discussed.

Other studies have reported the effect of the dimensions of piezoelectric ceramics [13] and the type of vibrations [14] on impact mode piezoelectric power generation. However, the effects of mechanical impact parameters on impact mode piezoelectric ceramic power generation are less discussed by researchers. Therefore, this study presents an analytical and experimental study on how to optimize the output power of impact mode piezoelectric power generators by analyzing two parameters that closely effect the output power: the velocity of impact and mass. To identify the relationship of the output power with these two parameters, a weight drop experiment was conducted. Variations in the weight of the objects and the height at which the objects were dropped, which is related to the impact velocity, were considered. The findings were then used to analyze the performance of a forced vibration-based impact mode piezoelectric power generator in harvesting vibration energy.

\section{Power Generation of the Piezoelectric Device in Impact Mode}

2.1. Piezoelectric in Impact Mode: Effects of the Impact Variables. Piezoelectric devices generate electricity through deformation of its structure. The cause of deformation can be vibration or direct impact on the structure. The characteristics of impact mode power generation of piezoelectric devices for various impact parameters can be studied by performing weight drop experiments. Theoretically, when an object with a weight of $m$ is dropped from a predetermined height, its impact force is given by the following:

$$
F=\frac{E_{k}}{h_{2}}=\frac{m v^{2}}{2 h_{2}}
$$

where $E_{k}$ is the kinetic energy, $h_{2}$ is the penetration distance, and $v$ is the velocity upon impact. Theoretically,
TABLE 1: Specifications of the round piezoelectric device.

\begin{tabular}{lc}
\hline Parameter & Value \\
\hline Diameter of metal plate & $35.0 \pm 0.1 \mathrm{~mm}$ \\
Diameter of ceramic element & $25.0 \pm 0.4 \mathrm{~mm}$ \\
Thickness of ceramic element & $0.21 \pm 0.05 \mathrm{~mm}$ \\
Young's modulus & $5.6 \times 10^{10} \mathrm{~N} / \mathrm{m}^{2}$ \\
Piezoelectric strain constant, $d_{33}$ & $420 \times 10^{-12} \mathrm{~m} / \mathrm{V}$ \\
Piezoelectric stress constant, $g_{33}$ & $23.3 \times 10^{-3} \mathrm{Vm} / \mathrm{N}$ \\
Capacitance, $C$ & $35 \mathrm{nF}$ \\
\hline
\end{tabular}

the maximum output energy per cycle of piezoelectric devices that operate in 33-mode is given by (2). From the equation, the amount of electrical energy that can be generated by a piezoelectric device is directly proportional to the square of the impact force:

$$
\begin{aligned}
& E_{\text {max }}=\frac{c}{a b} d_{33} g_{33} F^{2}, \\
& E_{\max }=\frac{c}{a b} d_{33} g_{33}\left(\frac{m v^{2}}{2 h_{2}}\right)^{2} .
\end{aligned}
$$

In this equation, $a, b$, and $c$ are the width, length, and thickness of the piezoelectric device, respectively, $d_{33}$ and $g_{33}$ are the piezoelectric charge (strain) and voltage (stress) constants, respectively, and $F$ is the force that acts on the device. Substituting (1) into (2) yields (3), which reveals that the output energy is directly proportional to $m^{2}$ and $v^{4}$ and inversely proportional to the penetration distance $h_{2}$. In drop-weight experiments, the two important impact variables are the weight and the velocity of the impact object. Assuming the object is dropped in a free fall and its height is $h_{1}$, the velocity of impact can be calculated using the equation $v=\sqrt{2 g h_{1}}$, where $g$ is the acceleration due to gravity.

2.2. Experiment and Discussion. The weight drop experimental setup is shown in Figure 1. The specifications of the piezoelectric device are listed in Table 1. The steel balls used in this experiment have diameters of $9.52 \mathrm{~mm}$ and $12.7 \mathrm{~mm}$ and weights of $4 \mathrm{~g}$ and $8 \mathrm{~g}$, respectively, and the material of the steel balls is carbon. Two types of supporting bases of the piezoelectric device are used: a flat base and a base with a hole. Two types of supporting bases are used to evaluate the output power with respect to the change in the stiffness. The diameter of the hole of the base is $30 \mathrm{~mm}$.

The experimental method consisted of dropping a steel ball in free fall from a predetermined height such that it hits the piezoelectric device. The output of the piezoelectric power generator was connected to load resistors of $1 \mathrm{k} \Omega, 10 \mathrm{k} \Omega$, and $20 \mathrm{k} \Omega$. The voltage is recorded to a data logger at a sampling time of $10 \mu \mathrm{s}$.

The experiment was conducted by varying the height of the steel ball from $10 \mathrm{~mm}$ to $70 \mathrm{~mm}$. When the steel ball was dropped, the piezoelectric device produced voltage pulses, as shown in Figure 2. For evaluation, only the first pulse was considered and compared. 


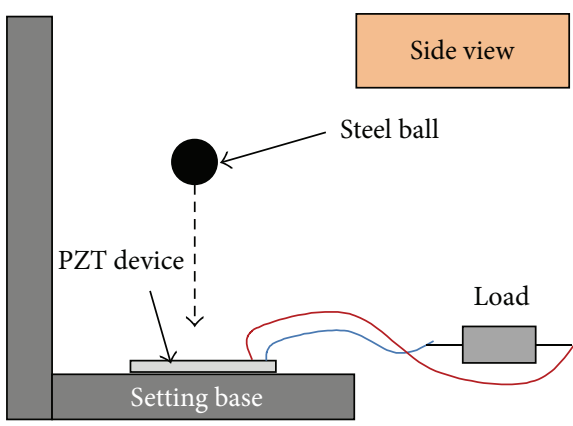

(a)

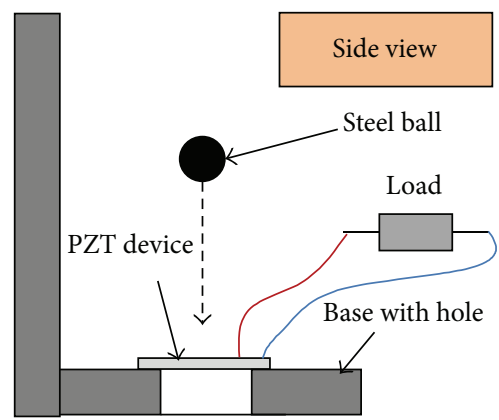

(b)

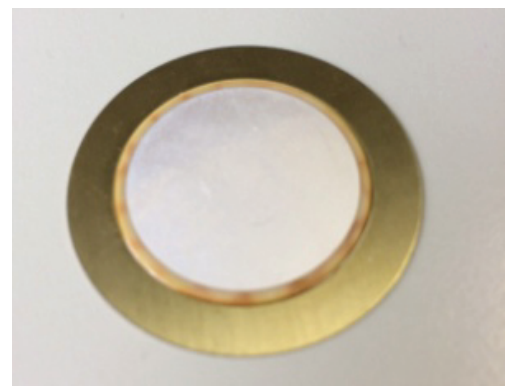

(c)

FIGURE 1: Experimental configuration: (a) flat base, (b) base with a hole, (c) a round piezoelectric device.

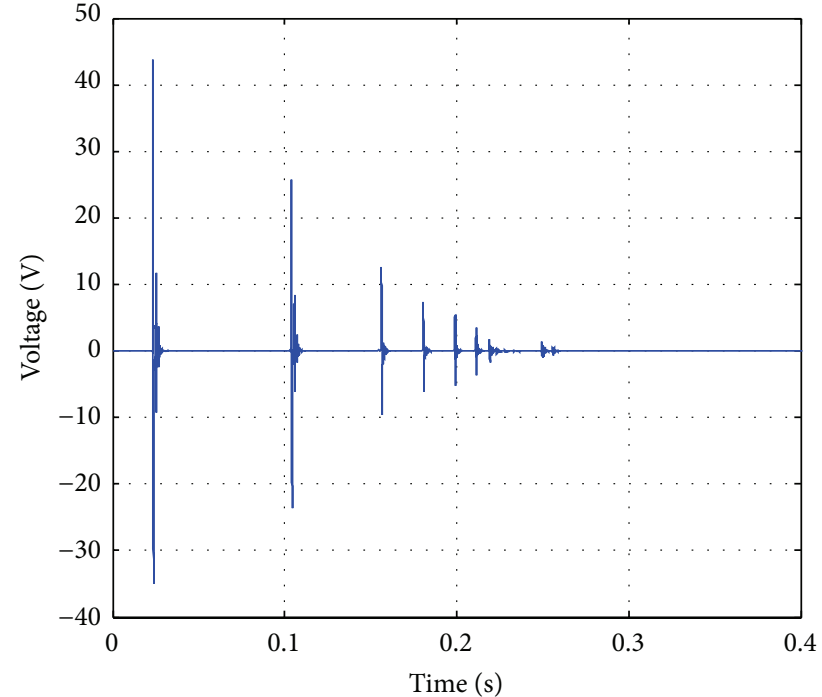

FIGURE 2: Example of the pulse signal.
Figure 3 shows the plot of the instantaneous peak output voltage versus the velocity of impact when the $4 \mathrm{~g}$ steel ball and the flat base were used. Regardless of the load, the instantaneous peak voltage is directly proportional to the velocity.

Meanwhile, output power of the load resistor $R$ is denoted by $P=V^{2} / R$. It is clear that power is proportional to the square of voltage $V$. As result, as illustrated in Figure 4, power is equal to the quadratic function of the velocity $v$. Here, we can relate the experimental results in Figures 3 and 4 with (1) and (2). As defined by (1), the impact force is also proportional to the square of the velocity $v$ and results in the power $P$ to be equal to the quadratic function of the force of impact $F$. This is supported by the experimental results in Figure 4. Moreover, the energy in (2) is the product of power and time. Therefore, the energy that can be generated by the piezoelectric device must equal to the quadratic function of the impact force $F$.

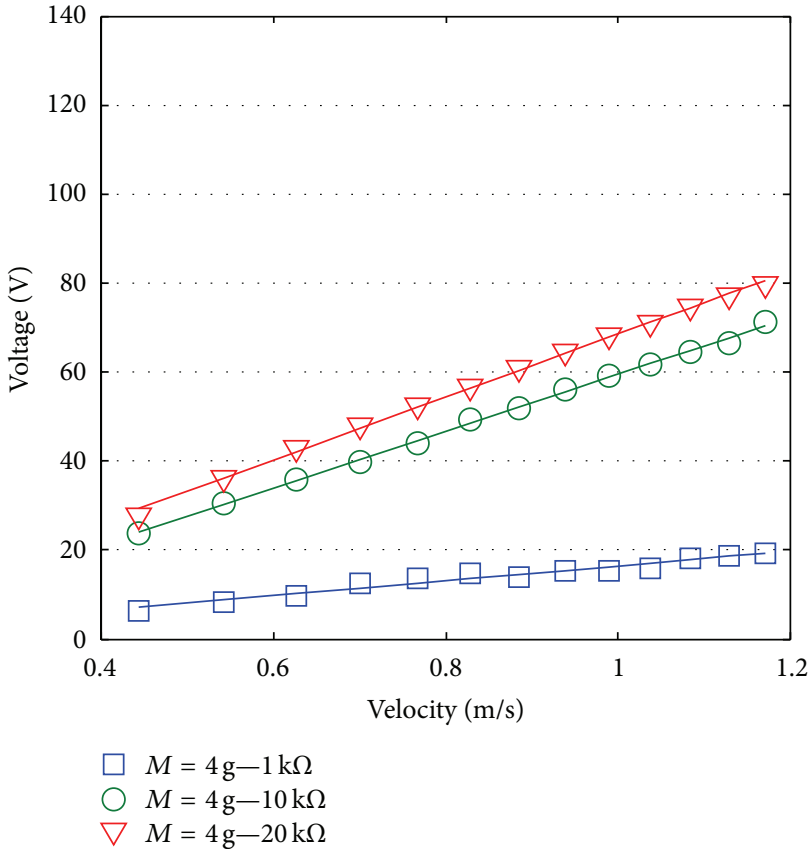

FIGURE 3: Instantaneous peak output voltage versus velocity.

Next, among the three load resistors, due to impedance matching, the output power of the $10 \mathrm{k} \Omega$ resistor corresponded to the highest average output power compared with the other load resistors.

For comparison and to observe the effect of the weight of the impact object on the output power of the piezoelectric power generator, the $4 \mathrm{~g}$ steel ball was replaced with an $8 \mathrm{~g}$ steel ball. As shown in Figure 5, for the same velocity, the output power of the heavier object generates higher output than the lighter object. For the same velocity, the heavier object possesses a higher impact force than the lighter object, as defined by (1). Therefore, heavier objects produce more power. However, when looking at the ball's momentum, as illustrated in Figure 6, for equal momentum, the output power of the lighter object is almost twice of that of the heavier object. 


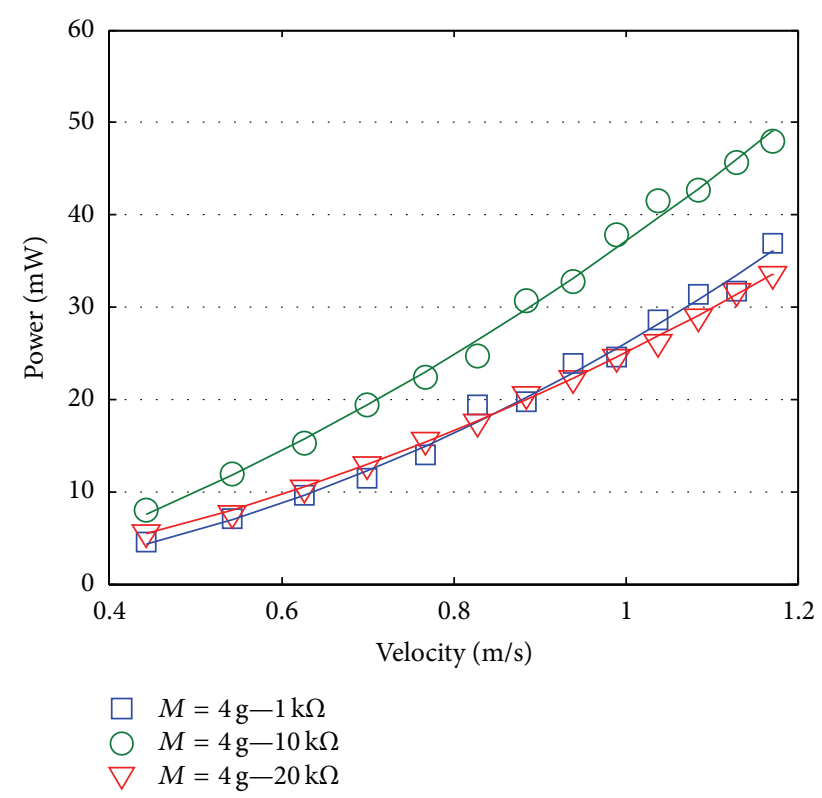

Figure 4: Average output power (2 ms) versus velocity.

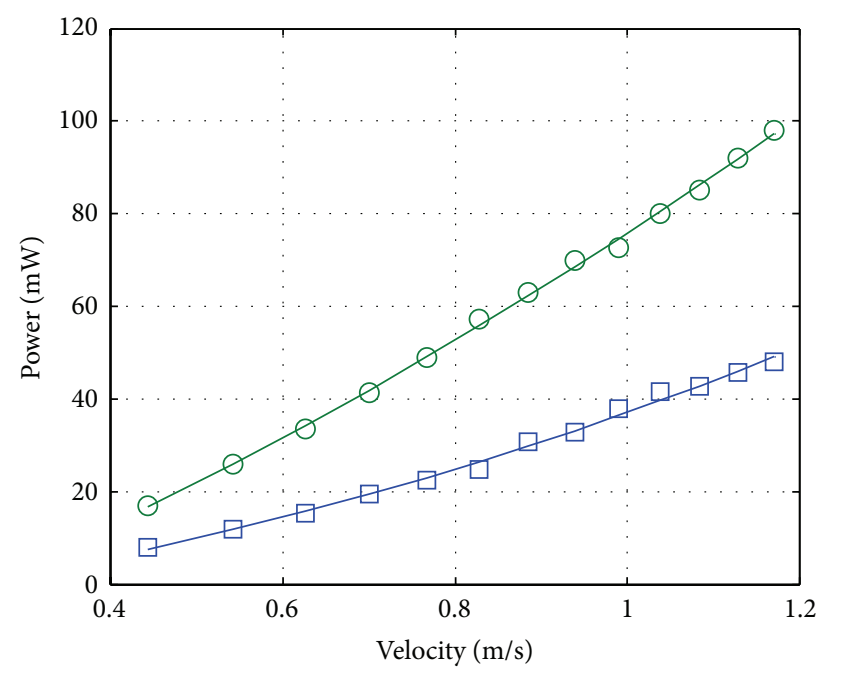

$\square M=4 \mathrm{~g}-10 \mathrm{k} \Omega$

$M=8 \mathrm{~g}-10 \mathrm{k} \Omega$

Figure 5: Average output power ( $2 \mathrm{~ms}$ ) versus velocity plots for $4 \mathrm{~g}$ and $8 \mathrm{~g}$ steel balls.

Therefore, for equal momentum, a higher average output power is obtained when using an object with a higher impact velocity rather than a heavier object.

Next, we evaluate the relationship between the output power and the stiffness of the piezoelectric device. The previous experiments were conducted using a piezoelectric device set on a flat iron base, which indirectly increased the stiffness of the device structure as a whole. In turn, incremental increases in the stiffness degraded the strain that developed on the piezoelectric device, resulting in lower output power. Therefore, to optimize the output power of the device, a base with a round hole was used to substitute

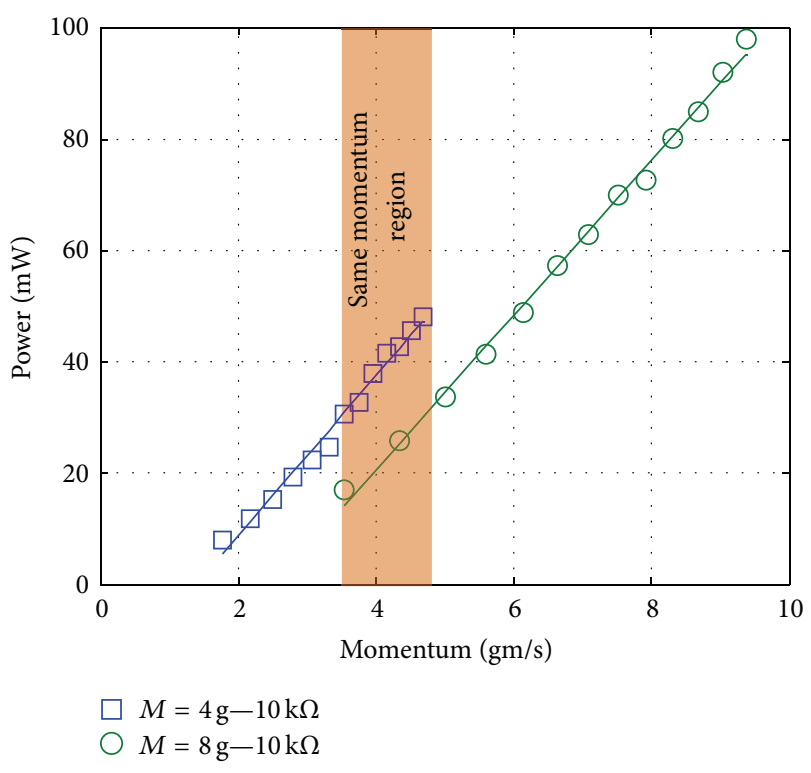

FIGURE 6: Average output power $(2 \mathrm{~ms})$ versus momentum plots for $4 \mathrm{~g}$ and $8 \mathrm{~g}$ steel balls.

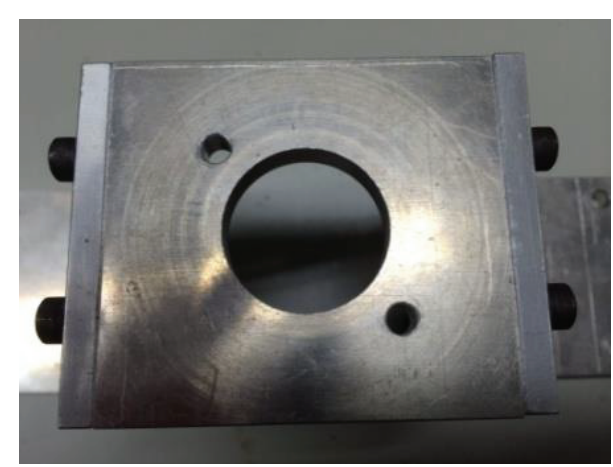

Figure 7: Iron base with hole.

the flat iron base. Figure 7 shows the base. The diameter of the hole is $30 \mathrm{~mm}$. The piezoelectric device was placed on the base with the proper adjustment such that the piezoelectric device is placed exactly on the hole.

The same weight drop experiments were conducted, varying the height and weight of the steel ball. The output power comparison is shown in Figure 8. In this figure, four datasets are plotted. It is obvious that when piezoelectric device was placed on the base with hole, its average output power has increased. For the highest momentum of $9.37 \mathrm{gm} / \mathrm{s}$, the difference in the output power was greater than $100 \mathrm{~mW}$. Therefore, increasing the stiffness of the entire structure of the piezoelectric device reduces the device efficiency. Thus, the stiffness of the device structure must be considered to improve efficiency.

\section{Device Configuration}

This section discusses the design and analyses of the vibration-based impact mode piezoelectric power generator. 


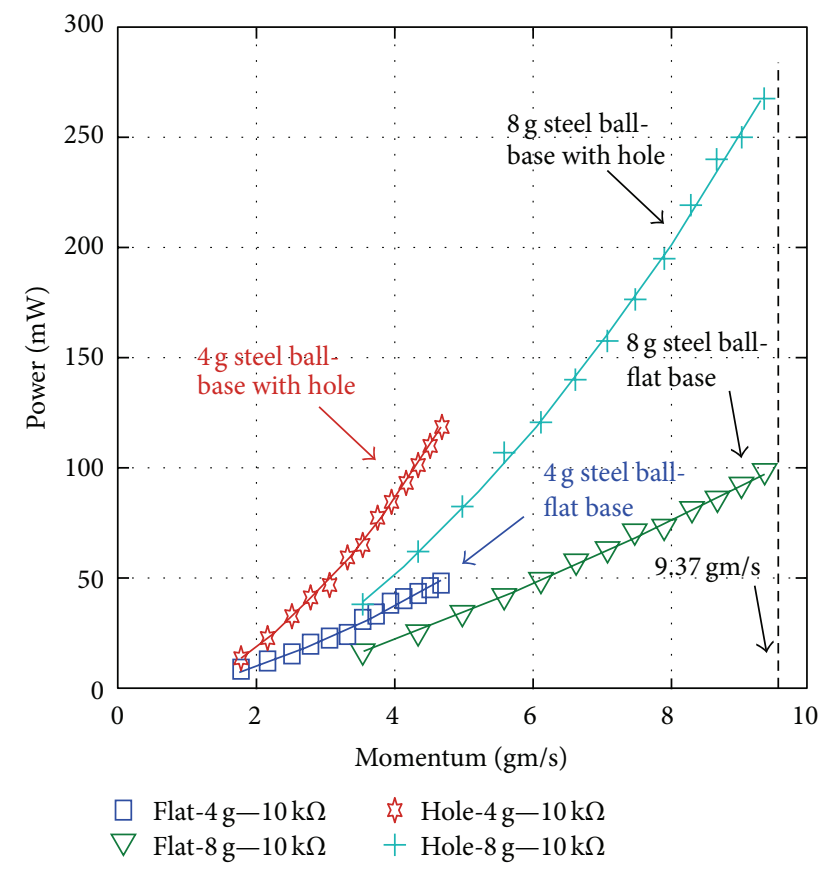

FIGURE 8: Average output power ( $2 \mathrm{~ms}$ ) versus momentum plots for $4 \mathrm{~g}$ and $8 \mathrm{~g}$ steel balls comparing the flat base and the base with a hole.

The configuration of the proposed power generator consists of three main structures: the base beam, an adjustable spacer, and the vibrating beam with the proof mass attached to the free end of the beam. Another important component attached to the free end of the vibrating beam is a metal tip. A schematic of the power generator is shown in Figure 9, and specifications of the structures are listed in Table 2. The thickness of the base beam is set to 10 times thicker than the vibrating beam to reduce antiresonances.

The operating principle of the power generator is to generate electricity from impact on the piezoelectric device. Every impact on the piezoelectric device produces a voltage pulse across the load resistor. The piezoelectric device is bonded to the base beam with epoxy and is placed on the hole of the beam so that as discussed in the previous section, the power generation can be optimized by maintaining the original stiffness of the piezoelectric device. The proof mass served as a deflection booster for the vibrating beam. Adjusting the weight of the proof mass is also performed for the resonant frequency variation. In addition, at the clamped area of the configuration, adjustable spacers were used to separate the vibrating beam and the base beam. The total thickness of the piezoelectric device is $0.41 \mathrm{~mm}$, and the height of the tip is $3 \mathrm{~mm}$. Based on a simple calculation, a $3 \mathrm{~mm}$ thickness spacer creates no gap between the tip and the piezoelectric device, thus allowing for a preloading condition on the piezoelectric device.

Increasing the thickness of the spacer to $4 \mathrm{~mm}$ should produce a gap between the tip and the piezoelectric device. However, the proof mass pressurizes the tip of the vibrating beam, which eventually results in the same situation as
TABLE 2: Specifications of the structures of the power generator.

\begin{tabular}{lc}
\hline Structure & Value \\
\hline $\begin{array}{l}\text { Base beam (aluminum) } \\
\text { Vibrating beam with hole } \\
\text { (aluminum) }\end{array}$ & $130 \times 50 \times 10 \mathrm{~mm}$ \\
$\begin{array}{l}\text { Adjustable spacer } \\
\text { (aluminum) }\end{array}$ & $100 \times 20 \times 1 \mathrm{~mm}$ \\
Proof mass (aluminum) & $26 \times 20 \times 1 \mathrm{~mm}$ \\
Round shape of tip (iron) & 26 and $40 \mathrm{~g}$ \\
& Height: $3 \mathrm{~mm}$ \\
& $\Phi: 4.5 \mathrm{~mm}$ \\
\hline
\end{tabular}

the $3 \mathrm{~mm}$ configuration. Although both configurations lead to a preloading condition on the piezoelectric device, in terms of the strength of the loads, the $3 \mathrm{~mm}$ configuration is expected to be higher. Thus, in comparing these two configurations, the $3 \mathrm{~mm}$ configuration requires a relatively higher velocity and frequency of vibration before the tip can vibrate and hit the piezoelectric device. Our analyses show that evaluation of the preloading condition on power generation from vibrations of frequencies below $100 \mathrm{~Hz}$ can be realized using a spacer thickness of $4 \mathrm{~mm}$. An additional $1 \mathrm{~mm}$ of spacer thickness creates a configuration with a gap between the tip and the piezoelectric device. Actual measurements show that the gap is approximately $0.604 \mathrm{~mm}$. Importantly, all of the above analyses were performed using a proof mass of $26 \mathrm{~g}$.

3.1. Piecewise Linear Model of the System. The amount of output power that can be generated is highly dependent on the number of impacts made by the tip. Generally, number of voltage pulses is directly proportional to the number of impacts. Thus, increasing the frequency of the vibrator will increase the number of impacts in one second. However, the impact velocity is dominant regarding the magnitude of the pulse. As discussed in the previous section, the peak of the output power is proportional to the square of the velocity. Thus, the output power generation for a period of time is expected to be dependent on the frequency of the vibrations and the velocity of the impact.

An evaluation of the motion of the vibrating beam is presented below using a piecewise linear model of the power generator as shown in Figure 10. The only part that is expected to vibrate is the system with a mass of $m$. This system represents the vibrating beam with the proof mass $m$ and the piezoelectric device with the spring constant $k_{1}$ and damping constant $c_{1}$. As previously mentioned, to prevent the base beam from deflecting and to reduce antiresonances, the thickness of the base beam was set to 10 times greater than the thickness of the vibrating beam. The behavior of impact is expected to be similar to an inelastic collision where the effective mass of the vibrating beam is negligible [15]. In this case, as the tip hits the piezoelectric device, it will stick to the device until their momentums become zero and then move back in the $x$ direction before separating from the device. As the tip engages the device, the damping constant and the spring constant become $c+c_{1}$ and $k+k_{1}$, respectively. Next, the vibrating beam vibrates freely until it reaches 


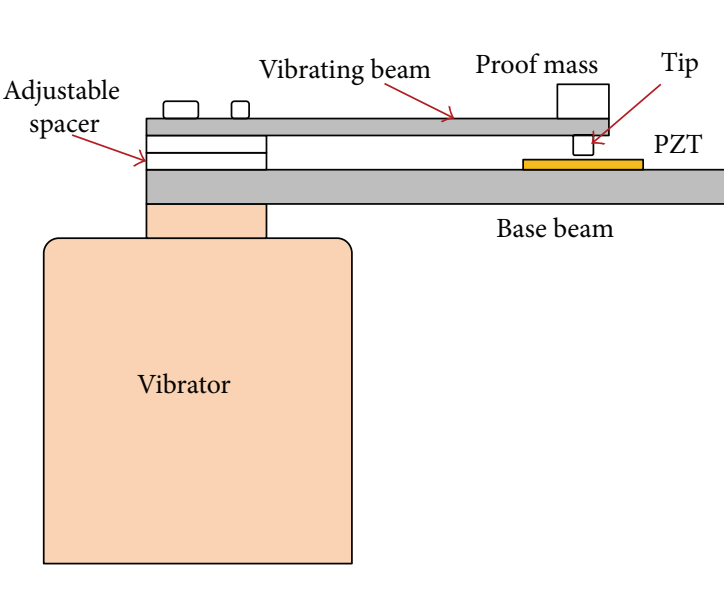

Construction of the power generator

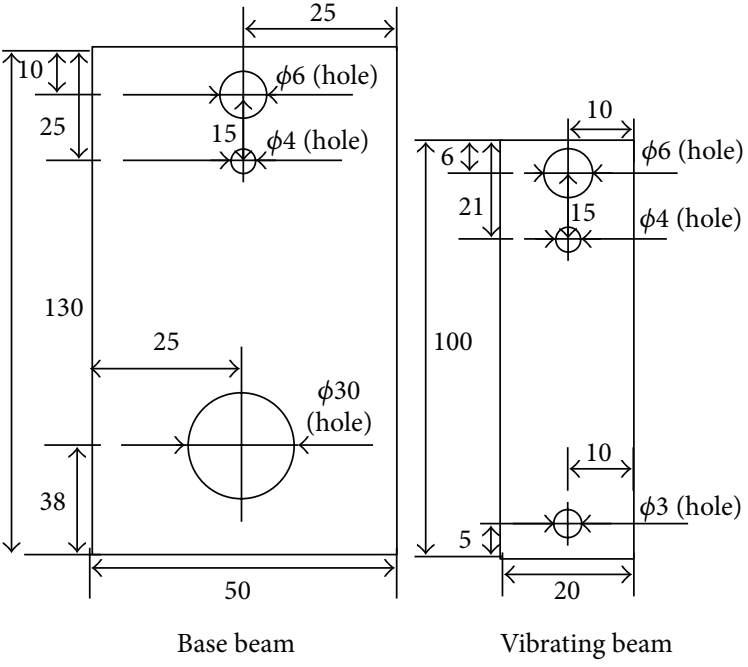

Base beam

Figure 9: Construction of the power generator and schematics of the structures.

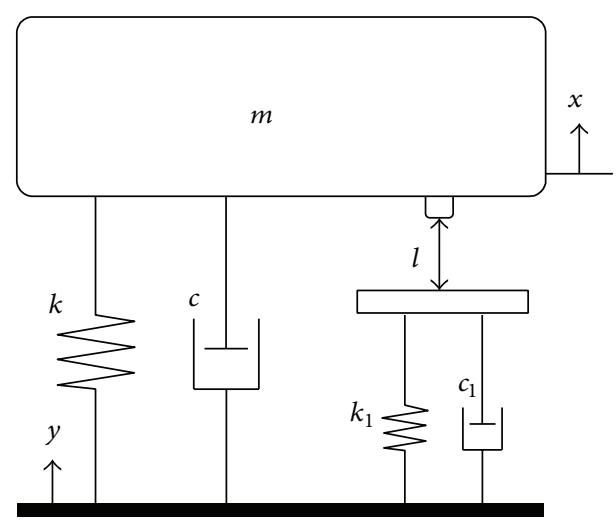

FIGURE 10: Piecewise linear model of the forced vibration-based impact mode piezoelectric power generator.

the maximum displacement and hits the device again. The system can be represented by the differential equation given in the following:

$$
\begin{aligned}
m \ddot{x}+c(\dot{x}-\dot{y})+k(x-y) & =0 \quad x>-l, \\
m \ddot{x}+\left(c+c_{1}\right)(\dot{x}-\dot{y})+\left(k+k_{1}\right)(x-y) & =0 \quad x<-l,
\end{aligned}
$$

where $y$ is the external excitation signal and $x$ is the displacement of the mass. In this study, $y(t)=Y_{0} \sin (\omega t)$, where $Y_{0}$ is the excitation amplitude and $\omega$ is the applied vibration's frequency. The detailed numerical solutions of these set of equations are reported in [16].

\section{Experimental Results and Discussion}

4.1. Experimental Setup and Conditions. The experimental setup is shown in Figure 11. The input signal to the vibrator and the output are monitored and recorded using a PC running the DSP-based controller software. The sampling time was set to $20 \mu \mathrm{s}$. The input signal voltage was fixed, whereas the vibration frequency was varied. Variations in the frequency cause the acceleration of the vibration signal to vary. Our analyses evaluated the power generation in the frequency range below $100 \mathrm{~Hz}$.

Based on the weight drop experiment results in the previous section, the optimum output power was generated with a load resistor of $10 \mathrm{k} \Omega$. Therefore, in this experimental evaluation, the same resistor was used as the load. To evaluate the effect of the weight of the proof mass on the output, two proof masses were used: $26 \mathrm{~g}$ and $40 \mathrm{~g}$. Based on the system identification experimental results when a proof mass of $26 \mathrm{~g}$ was used, the resonant frequency of the power generator without the base beam was $24 \mathrm{~Hz}$. When a proof mass of $40 \mathrm{~g}$ was used, the frequency decreased to $19 \mathrm{~Hz}$. Power generators with $4 \mathrm{~mm}$ and $5 \mathrm{~mm}$ spacer thicknesses were constructed and examined. The differences between the two configurations are described in the previous section.

4.2. Results. As the vibrating beam vibrates and the tip hits the piezoelectric device, a voltage pulse signal is produced across the load resistor. The number of pulses per second is dependent on the frequency of the vibration; that is, for a $35 \mathrm{~Hz}$ vibration, 35 voltage pulses are generated. A sample of the output voltage is shown in Figure 12. Figure 12(a) shows the output voltage when an input vibration of $35 \mathrm{~Hz}$ was used to excite the power generator.

Figure 12(b) shows a plot of the output power of one pulse. Two parts of the pulses for one impact can be observed. Deformation of the device is induced by the impact produced in the first part of the output pulse. As the tip and the device separate, the device vibrates for some time before coming to a stop, generating another output pulse, as shown in the plot. The output pulse appears to last for approximately $5 \mathrm{~ms}$ for every impact.

The frequency response of the power generator is shown in Figure 13. The plot shows the average output power for $5 \mathrm{~ms}$. We begin our discussion by focusing on the configuration plots under the preloading condition. In 


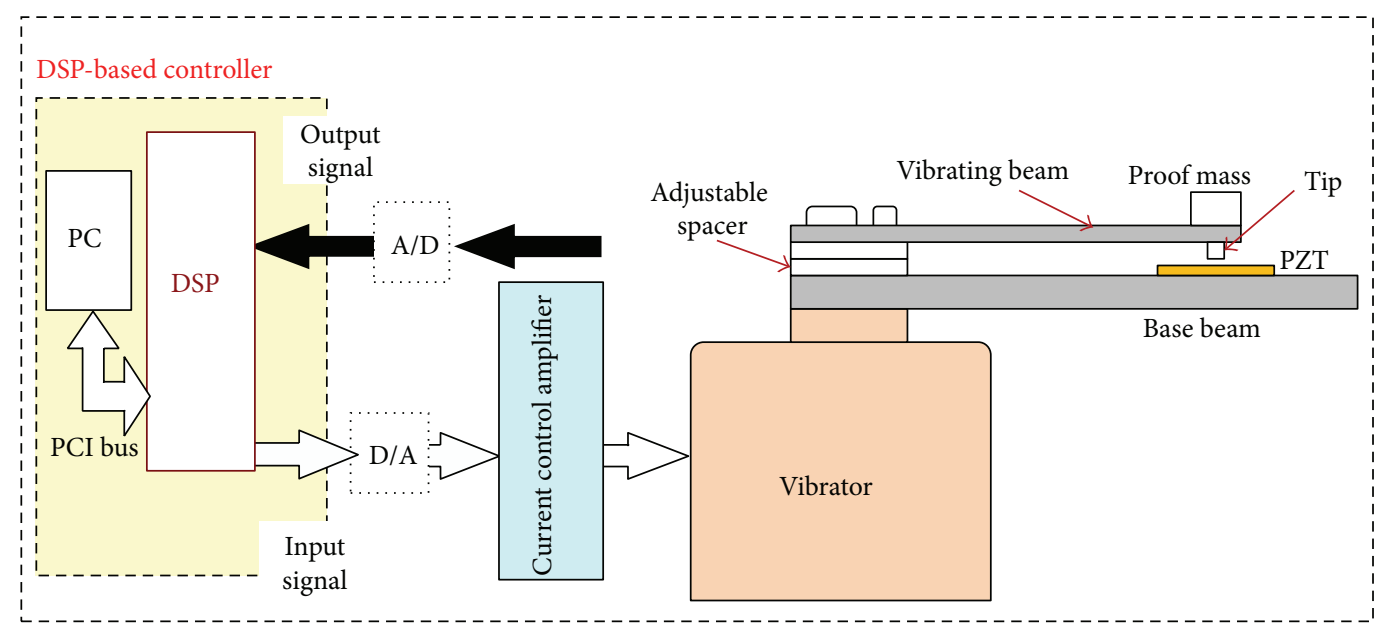

Figure 11: Experimental setup.

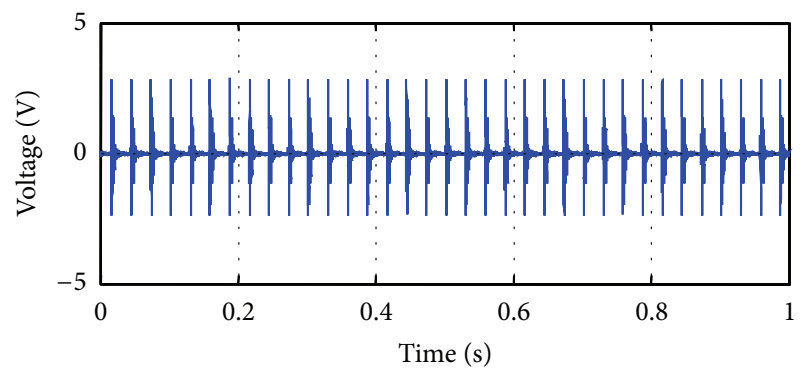

(a)

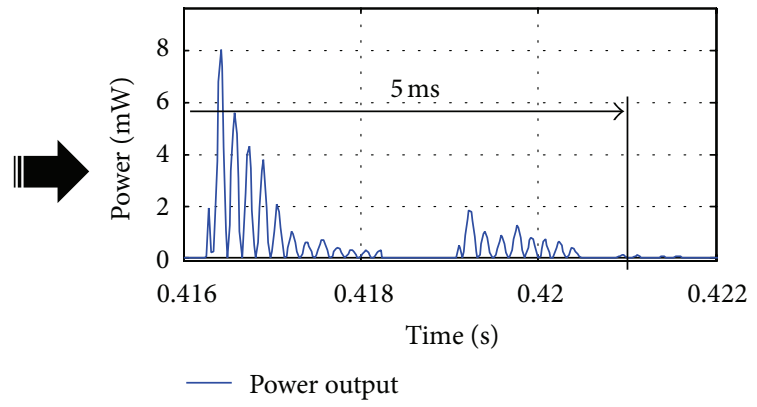

(b)

FIgURE 12: The output signals for a $35 \mathrm{~Hz}$ vibration, a spacer thickness of $4 \mathrm{~mm}$ and load resistor of $10 \mathrm{k} \Omega$. (a) The output voltage; (b) the output power.

the preloading condition, for which the spacer thickness is $4 \mathrm{~mm}$, the operating frequency bandwidth is wider than that of the configuration with a $5 \mathrm{~mm}$ spacer thickness regardless of the weight of the proof mass. The operating frequency bandwidth of the configuration with both proof masses is approximately $40 \mathrm{~Hz}$. Comparing these two plots, the magnitude of the configuration with the $26 \mathrm{~g}$ mass exhibited a peak output when the excitation frequency is approximately $39 \mathrm{~Hz}$. However, for the configuration with the $40 \mathrm{~g}$ proof mass, the excitation frequency peaked at $48 \mathrm{~Hz}$. The magnitude of the output power of the power generator with the lighter mass is relatively higher than that of the heavy mass. As previously discussed, the magnitude of the output power is proportional to the square of the impact velocity. Thus, for a lighter proof mass, the beam is expected to have a higher velocity upon impact. The significant difference in the magnitude is due to this factor. For a lighter proof mass, the tip begins to hit the device and generate output at lower frequencies. Due to the weight, the power generator with the heavy proof mass only begins to hit the device when the frequency of vibration is increased to approximately $35 \mathrm{~Hz}$. Thus, for the preloading condition, shifting the operating frequency of the power generator to a high frequency bandwidth can be simply achieved by increasing the proof mass weight. However, increasing the weight of the proof mass subsequently decreases the output magnitude.

For a power generator with a gap between the tip and the device, the operating frequency bandwidth decreases to approximately $25 \%$ of that of the configuration under the preloading condition. For a gap $0.604 \mathrm{~mm}$ between the tip and the device, the motion of the tip of the vibrating beam must be greater than that for power generation. Considering the vibration of the vibrator, increasing the vibration frequency beyond the resonant frequency of the power generator decreases the acceleration of the tip. Therefore, as the frequency reaches $33 \mathrm{~Hz}$ or $27 \mathrm{~Hz}$ for the $26 \mathrm{~g}$ and $40 \mathrm{~g}$ configurations, respectively, the motion of the tip becomes less than $0.604 \mathrm{~mm}$. From the same plot, operating frequency of the configuration with the lighter proof mass is slightly higher than the configuration with the heavier proof mass. This characteristic differs from the configuration in the preloading condition, as discussed earlier.

Furthermore, the results show that output power of the power generator for both configurations is very unstable. Our earlier assumption was that by setting the base beam to 10 times thicker than the vibrating beam, the occurrences of antiresonances of the response will reduce. However, as observed in the frequency response, the resonances are 


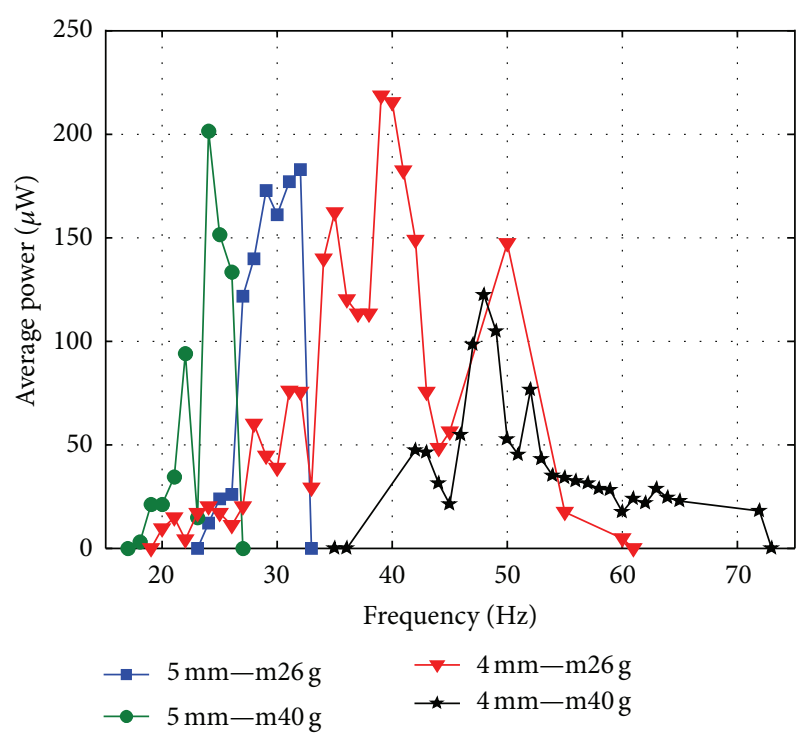

Figure 13: Plot of average output power ( $5 \mathrm{~ms}$ ) versus frequency.

separated by antiresonances, especially for the configuration under the preloading condition. To explain this phenomenon, we must analyze the motion of the structures of the power generator.

As discussed previously, the basic structure of the proposed power generator consists of a vibrating beam and a base beam, which are coupled at one end. When a vibration is applied to the structures, the base beam is ideally assumed to vibrate without deflection. In addition, a vibrating beam with a thickness of $1 \mathrm{~mm}$ and a proof mass attached will vibrate and deflect at the free end. Variations in the frequency will vary the amplitude of the free end of both beams. Therefore, a small change in the frequency subsequently changes the timing of the tip hitting the piezoelectric device on the base beam. The velocity of the impact severely varies with these changes, which contributes to the unstable output power over the operation frequencies. The resonance output is expected to be generated when the vibrating beam hits the piezoelectric device at its peak velocity as it crosses the equilibrium point. In addition, for the antiresonance output, the impact occurs at a low velocity of the vibrating beam.

\section{Conclusions}

Analytical and experimental studies of the effects of the mechanical impact parameters on piezoelectric power generation in impact mode are presented. The experimental data given in the first part of this study show that the velocity of impact affects the forces and subsequently the output power and the energy to a greater degree than the mass. Moreover, it was experimentally demonstrated that, the optimum output power can be achieved if the stiffness of the device is maintained at its original value so that the efficiency does not decrease. In the second part of this study, analyses of the output power of the vibration-based impact mode power generator were presented. Two configurations were analyzed. Although the operating frequency of the preload configuration is wider than the configuration with the gap, due to the relative motion of the two beams, the output power suffers the resonance and antiresonance phenomena. Further evaluations of the structures of power generators should be performed in the future so that a stable output power and wider operating frequency bandwidths can be achieved.

\section{Conflict of Interests}

The authors declare that there is no conflict of interests regarding the publication of this paper.

\section{References}

[1] Z. Chen, B. Guo, Y. Luo, and Y. Yang, "Numerical investigations into the effects of multiple parameters on nonlinear piezoelectric vibration energy harvesters," Advances in Mechanical Engineering, vol. 6, Article ID 604704, 9 pages, 2014.

[2] S.-M. Chen and J.-H. Hu, "Experimental study of a hybrid vibration energy harvesting mechanism," in Proceedings of the Symposium on Piezoelectricity, Acoustic Waves, and Device Applications (SPAWDA '11), pp. 56-59, December 2011.

[3] Z. Wang, B. Wang, M. Wang, H. Zhang, and W. Huang, "Model and experimental study of permanent magnet vibration-toelectrical power generator," IEEE Transactions on Applied Superconductivity, vol. 20, no. 3, pp. 1110-1113, 2010.

[4] Y. Sang, X. Huang, H. Liu, and P. Jin, "A vibration-based hybrid energy harvester for wireless sensor systems," IEEE Transactions on Magnetics, vol. 48, no. 11, pp. 4495-4498, 2012.

[5] L. Yan, J. Hou, Z. Yang, and X. Chu, "Design and experimental characterization of a vibration energy harvesting device for rotational systems," Advances in Mechanical Engineering, vol. 2013, Article ID 263614, 7 pages, 2013.

[6] A. Shebeeb and H. Salleh, "Effect of cantilever shape on the power output of a piezoelectric bimorph generator," in Proceedings of the IEEE International Conference on Semiconductor Electronics, pp. 275-278, June 2010.

[7] A. A. Basari, S. Awaji, S. Wang et al., "Shape effect of piezoelectric energy harvester on vibration power generation," Journal of Power and Energy Engineering, vol. 2, no. 9, pp. 117-124, 2014.

[8] A. A. Basari, S. Awaji, S. Hashimoto et al., "Comparison and evaluation of vibration-based piezoelectric power generators," in Proceedings of the International Power Electronics Conference (IPEC-Hiroshima-ECCE-ASIA '14), pp. 3194-3199, Hiroshima, Japan, May 2014.

[9] N. Kong, D. S. Ha, A. Erturk, and D. J. Inman, "Resistive impedance matching circuit for piezoelectric energy harvesting," Journal of Intelligent Material Systems and Structures, vol. 21, no. 13, pp. 1293-1302, 2010.

[10] E. Simon, Y. Hamate, S. Nagasawa, and H. Kuwano, "3D vibration harvesting using free moving ball in PZT microbox," in Proceedings of the Power MEMS, pp. 33-36, 2010.

[11] M. A. Halim, S. Khym, and J. Y. Park, "Impact based frequency increased piezoelectric vibration energy harvester for human motion related environments," in Proceedings of the 8th Annual IEEE International Conference on Nano/Micro Engineered and Molecular Systems (NEMS '13), pp. 949-952, IEEE, Suzhou, China, April 2013.

[12] M. Han, Y. C. Chan, W. Liu, S. Zhang, and H. Zhang, "Low frequency PVDF piezoelectric energy harvester with combined 
d31 and d33 operating modes," in Proceedings of the 8th Annual IEEE International Conference on Nano/Micro Engineered and Molecular Systems (IEEE NEMS '13), pp. 440-443, April 2013.

[13] X.-R. Chen, T.-Q. Yang, W. Wang, and X. Yao, "Vibration energy harvesting with a clamped piezoelectric circular diaphragm," Ceramics International, vol. 38, pp. S271-S274, 2012.

[14] M. Okayasu, D. Sato, Y. Sato, M. Konno, and T. Shiraishi, "A study of the effects of vibration on the electric power generation properties of lead zirconate titanate piezoelectric ceramic," Ceramics International, vol. 38, no. 6, pp. 4445-4451, 2012.

[15] M. Renaud, P. Fiorini, R. Schaijk, and C. Hoof, "Harvesting energy from the motion of human limbs: the design and analysis of an impact based piezoelectric generator," Smart Materials and Structures, vol. 18, no. 3, Article ID 035001, 16 pages, 2009.

[16] A. Narimani, M. F. Golnaraghi, and G. N. Jazar, "Frequency response of a piecewise linear vibration isolator," Journal of Vibration and Control, vol. 10, no. 12, pp. 1775-1794, 2004. 

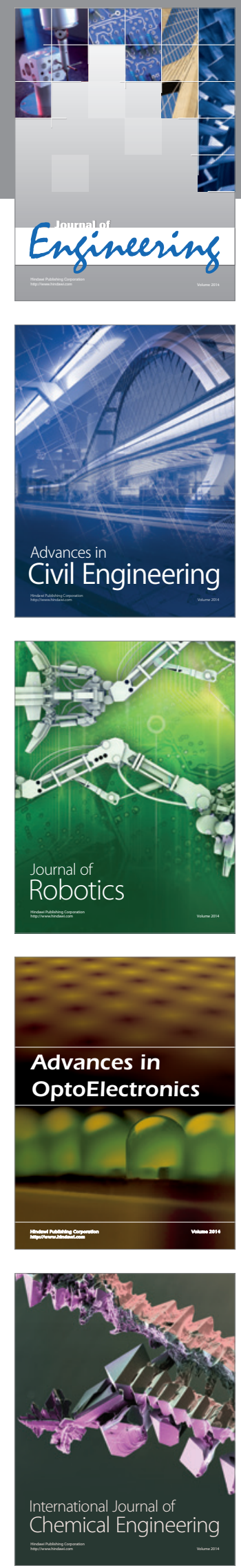

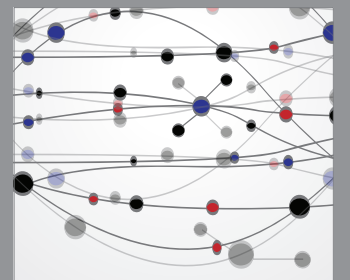

The Scientific World Journal
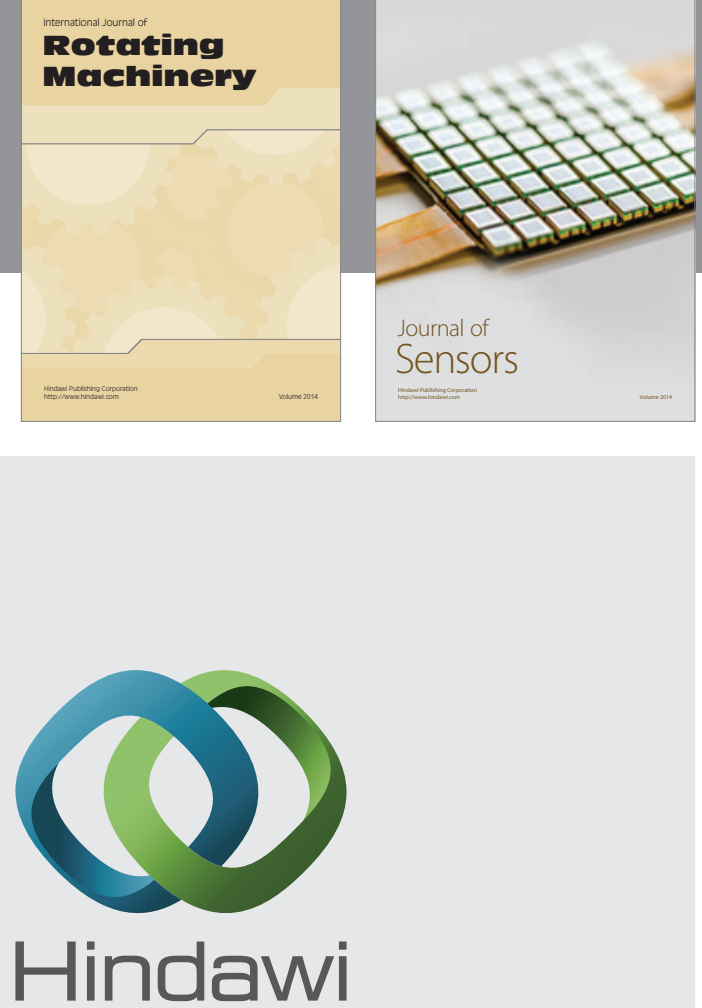

Submit your manuscripts at http://www.hindawi.com
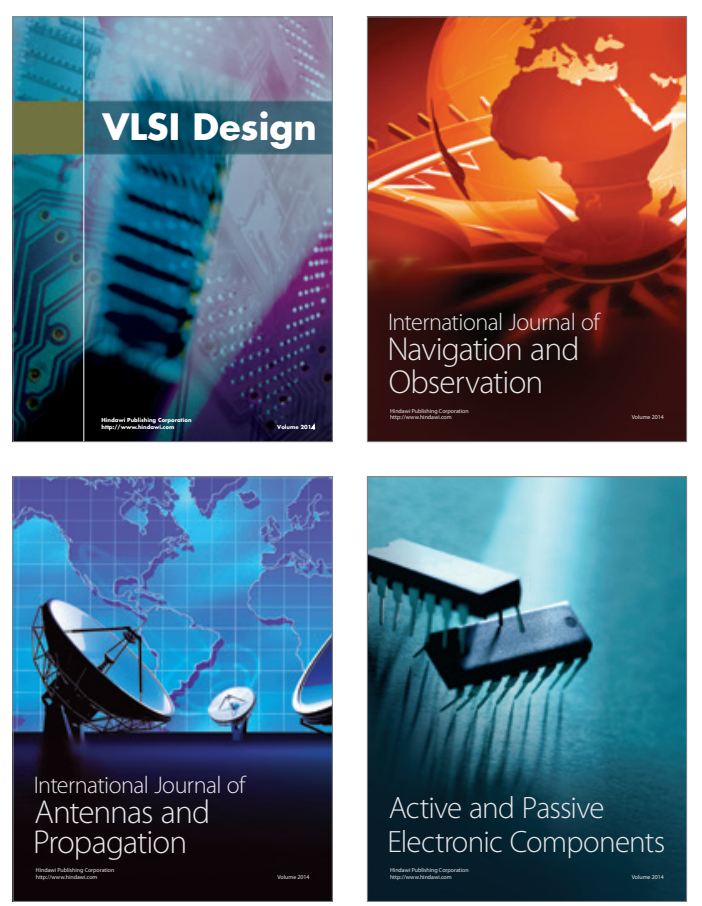
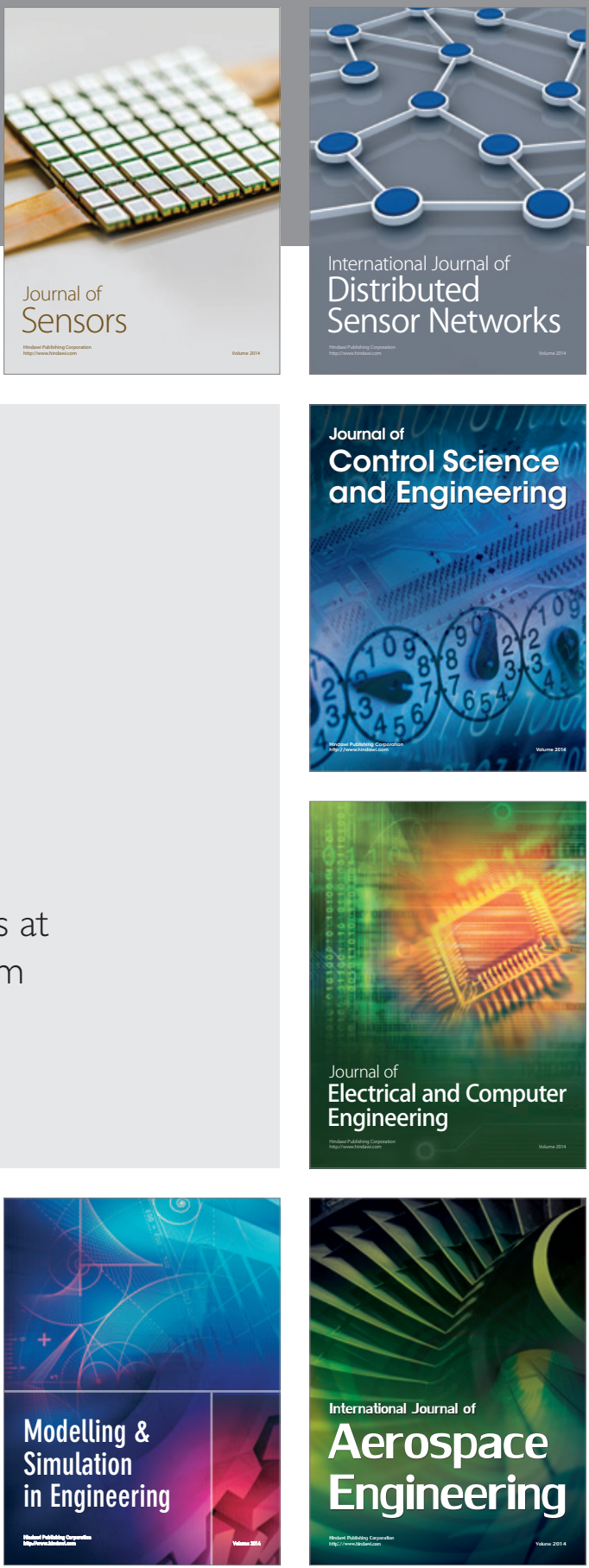

Journal of

Control Science

and Engineering
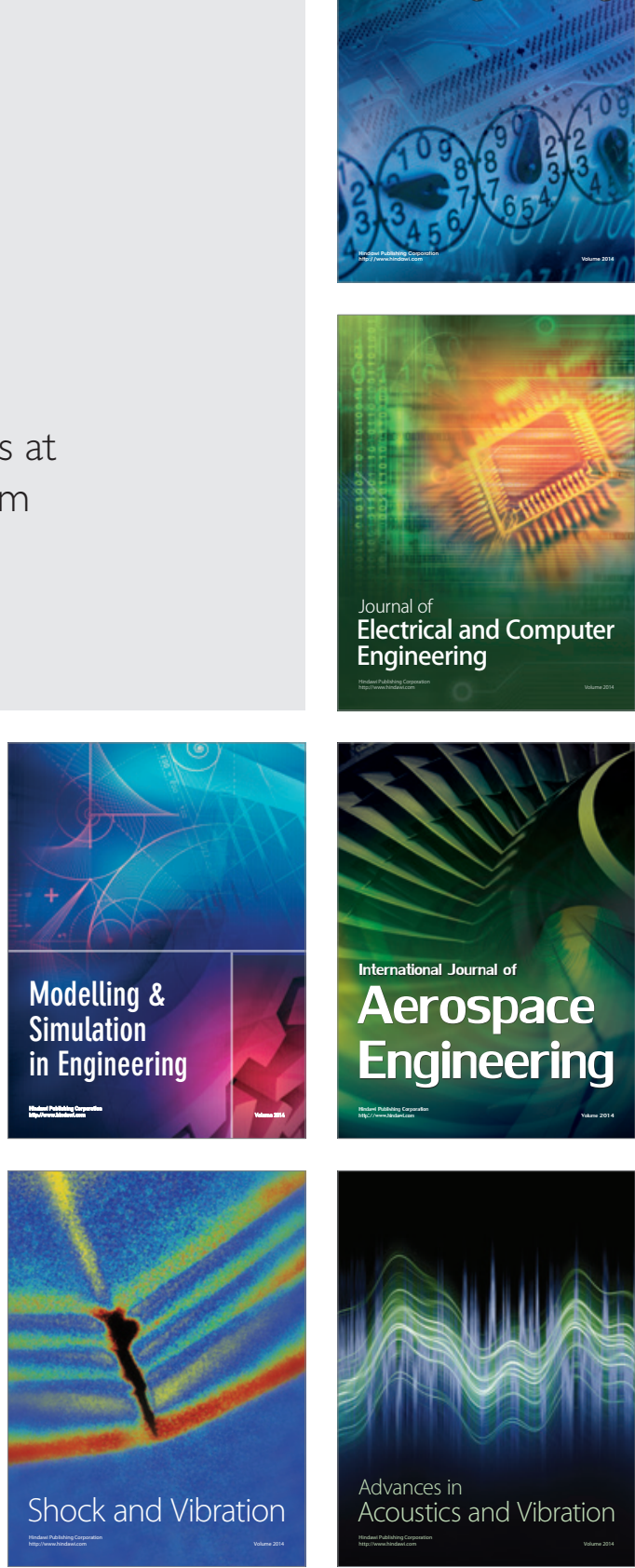